\title{
DOPPLERVELOCIMETRIA DO DUCTO VENOSO NA PREDIÇÃO DA ACIDEMIA FETAL
}

\author{
Francisco Herlânio C. Carvalho*, Antonio Fernandes Moron, Rosiane Mattar, Renato Martins Santana, Carlos Geraldo V. Murta, \\ Maurício Mendes Barbosa, Maria Regina Torloni, luiz Kulay Junior \\ Trabalho realizado no Hospital São Paulo - Universidade Federal de São Paulo - Escola Paulista de Medicina, São Paulo, e na \\ Maternidade-Escola Assis Chateaubriand - Universidade Federal do Ceará, Fortaleza, Ceará
}

\footnotetext{
*Correspondência:

R. Osvaldo Cruz, 2582

Ap. 202, Dionísio

Torres, Fortaleza, CE,

Cep 60125-151,

Telefone: (85) 458-2121

herlaniocosta@uol.com.br
}

\section{RESUMO}

OBjetivos. Investigar a possibilidade da predição da acidemia no nascimento mediante dopplervelocimetria do ducto venoso e definir qual o melhor parâmetro e seus pontos de corte nessa predição em gestações com insuficiência placentária.

Métodos. Trata-se de estudo transversal e prospectivo que analisou 47 gestaçooes únicas com insuficiência placentária e idade gestacional superior a 26 semanas, realizado no Hospital São Paulo (UNIFESP) e na Maternidade-Escola Assis Chateaubriand (UFC). A insuficiência placentária foi diagnosticada quando o índice de pulsatilidade da artéria umbilical encontrava-se acima do percentil 95 para a idade gestacional estimada. Fetos com anomalias estruturais ou cromossômicas foram excluídos. 0 doppler foi realizado a menos de 24 horas do parto. A amostra de sangue da artéria umbilical foi coletada imediatamente após o nascimento para análise da gasometria. Diagnosticou-se acidemia quando 0 pH encontrava-se abaixo de 7,20 na ausência de trabalho de parto e abaixo de 7,15 quando parto vaginal. Foram consideradas patológicas as acidemias metabólicas ou mistas. Construiu-se curva ROC para as velocidades S, D e A e para 0 IPV e as relações $S / A$ e (S-A)/S do DV (variáveis independentes) e acidemia (variável dependente). 0 teste de MacN emar foi utilizado para comparar os parâmetros entre si.

Resultados. As velocidades absolutas $S$, D e A mostraram ser pobres preditoras da acidemia no nascimento. 0 IPV mostrou ser bom preditor de acidemia (área sob a curva ROC $0,79, p=0,003$ ). As relações $S / A$ e $(S-A) / S$ também mostraram ser boas preditoras da acidemia (área sob a curva ROC $0,818, p=0,001$ ). Os pontos de corte calculados foram: $\mathbb{I P V}=0,76$, $S / A=2,67$ e $(S-A) / S=0,63$.

Conclusöess. Os índices ângulo-independentes do doppler do DV mostraram excelente correlação com acidemia no nascimento nesta população. Não houve diferença estatisticamene significativa entre estes parâmetros.

Unitermos: Dopplervelocimetria. Ducto venoso. Acidemia fetal.

\section{INTRODUÇÃO}

0 advento da dopplervelocimetria nas três últimas décadas permitiu 0 estudo da hemodinâmica útero e feto-placentária por meio da análise das artérias uterinas e umbilicais, e, proporcionou melhoria nos resultados perinatais. Portanto, é a metodologia preferida na atualidade para o diagnóstico e seguimento das gestações com insuficiência placentária1,2 .

A aplicação da dopplervelocimetria foi introduzida na obstetrícia por Fitzgerald e D rumm, em 1977, com a análise do fluxo na artéria umbilical. Posteriormente, com o aprimoramento dos aparelhos de ultra-sonografia, a avaliação foi estendidaà circulação fetal propriamente dita e, na última década, o território venoso fetal passou a ser desvendado. A partir daí, os fenômenos adaptativos fetais, estudados no passado por meio de modelos hipoxêmicos em animais, puderam ser demonstrados na espécie humana.

As alterações hemodinâmicas são desencadeadas pelo estímulo dos quimiorrecepto res quando o feto é exposto a condições pobres em oxigênio. 0 mecanismo adaptativo visa proteger os órgãos considerados nobres, cujas funções devem ser preservadas para a manutenção das atividades vitais do feto. U m maior fluxo de sangue é destinado ao sistema nervoso central, miocárdio e glândulas adrenais. Esse privilégio ocorre em detrimento dos demais órgãos, nos quais ocorre vasoconstrição (fenômeno denominado centralização da circulação fetal). N essa fase, observam-se anormalidades no segmento arterial fetal ${ }^{3,4}$.

Em situações de grave comprometimento placentário, os mecanismos compensatórios alcançam seus limites e a oxigenação do miocárdio torna-se insuficiente para proporcionar adequada contratilidade e uma efetiva ejeção do sangue. A queda no débito cardíaco é atribuída à miocardiopatia hipoxêmica com subseqüente disfunção ventricular 3,5 .

A descompensação hemodinâmica é clinicamente reconhecida pelo padrão anormal do doppler venoso. Com a queda no débito cardíaco, a pressão venosa central eleva-se causando aumento no fluxo reverso durante sístole atrial na veia cava inferior e surgimento de fluxo zero ou reverso no ducto venoso (DV). A progressão dos padrões de fluxos anormais nas veias precordiais reflete, presumivelmente, estágios progressivos da disfunção miocárdica e sua correlação com a gravidade da acidemia ${ }^{3,5}$.

A transição entre a adaptação e a descompensação devida à hipoxemia é difícil de ser identificada acuradamente. Não existe, na atualidade, intervenção terapêutica efetiva capaz de reverter 0 curso progressivo da insuficiência placentária, exceto a resolução da gestação. 0 timizar a assistênciae decidir o momento da intervenção é complexo, 
pois requer a comparação dos riscos da prematuridade contra aqueles da permanência intra-uterina: morte e lesão de múltiplosórgãos devido à inadequada perfusão tissular ${ }^{6}$. U ma conduta razoável é permitir a manutenção da gestação até o ponto anterior à lesão fetal, portanto minimizando tanto os riscos da prematuridade quanto da própria lesão orgânica. A questão clínica é saber que ponto seria esse e como 0 seguimento pode ser realizado.

0 doppler da artéria umbilical é verdadeiramente um teste para análise da função placentária que permite a identificação dos fetos de risco para resultados adversos, mas é um pobre preditor da condição fetal. Em contraste, 0 doppler venoso nos permite definir a severidade dos resultados perinatais, pois descreve o grau do comprometimento circulatório do feto 4,7 .

Há evidências crescentes de que o doppler pode ter papel central nas decisões perinatais, avaliando o risco de lesões teciduais ou morte intra-uterina comparado aos riscos da prematuridade nesses fetos comprometidos ${ }^{7,8}$. Desde que se conhece a seqüência de alterações, múltiplos vasos (artéria umbilical, artéria cerebral média e veias precordiais) devem ser avaliados, mas 0 DV oferece uma combinação de vantagens: é um regulador importante do retorno venoso tanto em fetos normais quanto anormais, é um canal direto para transmissão das ondas de pulso retrógrado do átrio direito, é responsivo a variações na concentração de oxigênio independente da função cardíaca e é identificável com relativa facilidade devido a sua alta velocidade de fluxo com o doppler colorido 4.

0 DV parece ser, no momento, o melhor vaso para monitorização de fetos comprometidos colaborando nadecisão sobre o momento da resolução da gestação em virtude de se traduzir numa alteração não tão precoce quanto o doppler arterial, nem tão tardia como anormalidades na veia umbilical ou nas outras provas biofísicas 4, .

Ainda há resultados diferentes quanto ao parâmetro no doppler do DV que melhor traduz o risco de comprometimento fetal: análise qualitativa com velocidade durante sístole atrial zero ou reversa3,4,10,11, índices ângulo-independentes acima do percentil $95^{12,13}$, velocidade $A$ abaixo do percentil $5^{12}$, relação $S / A$ maior que $3,6^{14}$ ou velocidade durante contração atrial $<17 \mathrm{~cm} / \mathrm{s}$, índice de pulsatilidade para veias $(\mathrm{IPV})>0,90$ e $S-\mathrm{A} / \mathrm{S}>0,70^{15}$.

0 objetivo do presente estudo foi investigar a probabilidade de predição de acidemia fetal através das velocidades $(S, D$ e $A)$ e índices ângulo-independentes (IPV, S/A e S-A/S) no doppler do DV e definir qual o melhor parâmetro e seu ponto de corte nessa determinação em gestações com insuficiência placentária.

\section{MÉTODOS}

Trata-se de estudo transversal e prospectivo com análise do doppler do ducto venoso em gestações com insuficiência placentária, realizado no Hospital São Paulo (U niversidade Federal de São Paulo Escola Paulista de Medicina) e na Maternidade-Escola Assis C hateaubriand (U niversidade Federal do C eará). A coleta de dados foi realizada entre 1 de março de 2001 e 31 de outubro de 2003.

A presente pesquisa atendeu aos preceitos da Declaração de Helsinque, emendada em Hong-Kong, e segue ostermos preconizados pelo Conselho N acional de Saúde (portaria 196/1996) para pesquisa com seres humanos. Todas as pacientes foram informadas sobre os objetivos da pesquisa, os exames a serem realizados e sua segurança, e, após esclarecimento das dúvidas, assinaram o Termo de Consentimento Livre e Esclarecido. 0 protocolo de estudo foi aprovado pela Comissão de Ética em Pesquisa de ambas as instituições.

0 estudo incluiu 47 pacientes com diagnóstico de insuficiência placentária (presença de índices de resistência e pulsatilidade na artéria umbilical acima do percentil 95 para a idade gestacional estimada, calculados pelas tabelas de Arduini e Rizzo,199016), persistência de incisura protodiastólica nas artérias uterinas, gestações únicas, idade gestacional > 26 semanas, ausência de anomalias estruturais ou cromossômicas no feto ou RN , último exame de doppler realizado a menos de 24 horas do parto, RN vivo, gasometria realizada no cordão umbilical no momento do parto e parto realizado em uma das duas instituições incluídas no estudo. Foram excluídas as gestantes com diagnóstico de descolamento prematuro de placenta antes ou durante o parto, anestesia geral quando parto abdominal ou que se recusaram a prosseguir no estudo.

Q uinze pacientes foram selecionadas no setor de patologia obstétrica no Hospital São Paulo e 32 na Maternidade-Escola Assis Chateaubriand.

Treze pacientes não apresentavam patologias associadas com a insuficiência placentária. As patologias associadas às outras 34 pacientes foram: 17 doença hipertensiva específica da gestação (DHEG) na sua forma pura (destas, uma apresentou eclâmpsia e a outra síndrome HELLP), dez apresentavam hipertensão arterial crônica (duas delas evoluíram com DHEG superajuntada), duas portadoras da síndrome do anticorpo antifosfolípide, duas do vírus HIV, uma diabética insulino-independente, uma cardio patia reumática e uma portadora de carcinoma mamário que realizou quimioterapia durante a gestação.

A idade média da população foi de 28,9 anos (variando de 16 a 44 anos). A maioria tratava-se de pacientes multíparas (28 pacientes $=60,6 \%)$. A idade gestacional média no momento do parto foi de 32,9 semanas (variando de 26 a 40 semanas). A gestação foi resolvida pela cesárea em 42 pacientes $(89,4 \%)$ e houve cinco partos vaginais $(10,6 \%)$. 0 s recém-nascidos pesaram, em média, $1.581 \mathrm{~g}$ (variando de 500 a $2.730 \mathrm{~g}$ ). Q uanto à adequação do peso à idade gestacional, 42 RN foram classificados como PIG $(89,4 \%)$ e 5 como AIG $(10,6 \%)$. Trinta e do is recém-nascidos $(68,1 \%)$ apresentaram Apgar no primeiro minuto maior ou igual a 7 e 15 deles menor que 7. No quinto minuto, 44 dos recém-nascidos $(93,6 \%)$ mostravam Apgar maior ou igual a 7.

A idade gestacional foi estimada pela data da última menstruação, desde que conhecida e confiável, e confirmada por exame ecográfico, realizado antes de 20 semanas. Pacientes com datadaúltimamenstruação desconhecida ou pouco confiável foram admitidas no estudo quando havia a informação de exame ecográfico precoce. N esse caso, a idade gestacional foi corrigida pela ultra-sonografia.

0 tamanho da amostra foi calculado com o auxílio do programa EPI IN FO. Fixando um erro alfa de $5 \%$ (confiança de $95 \%$ ), um erro beta de $10 \%$ (poder do estudo de $90 \%$ ) e considerando-se uma freqüência esperada de acidemia no cordão umbilical ao nascimento em torno de $20 \%$ no grupo de gestações de alto risco, foi encontrado "n" total de 34 pacientes. 
O s exames foram realizados em equipamentos de ultra-som das marcas Dysonic (GE) ou Siemens Versa-Pro com capacidade para estudo dopplervelocimétrico co lo rido e pulsátil. U tilizou-se transdutor convexo de $3,5 \mathrm{MHz}$. As medidas dopplervelocimétricas foram obtidas durante período de repouso, na ausência de movimentos respiratórios fetais ou de contrações uterinas. 0 ângulo de insonação foi considerado satisfatório quando $<30^{\circ}$ na análise dos vasos arteriais e sempre de 0 grau na avaliação do DV, pois se analiso u as velocidades específicas no cálculo dos índices. Utilizou-se filtro de $100 \mathrm{~Hz}$, para minimizar as chances de erros causados pelo movimento das paredes vasculares. 0 tamanho da amostra de volume foi ajustado de acordo com 0 diâmetro do vaso analisado; em particular para o DV, utilizou-se janela de 1 a $2 \mathrm{~mm}$. As medidas verificadas foram efetuadas após a obtenção de pelo menos três ondas seguidas e simétricas.

Durante a realização do exame dopplervelocimétrico, as pacientes eram acomodadas em posição semi-Fowler, objetivando evitar a hipotensão postural. Todos os exames foram realizados pelo mesmo pesquisador (FHCC).

Como local de amostragem para obtenção do sonograma da artéria umbilical adotou-se a sua porção mais distal (próximo à sua inserção placentária). 0 segmento escolhido era selecionado pelo doppler colorido e posteriormente acionado o doppler pulsátil para obtenção das ondas de velocidade de fluxo. 0 fluxo da veia umbilical deveria ser visualizado no mesmo corte.

Foram determinados os índices de resistência $(A-B / A)$ e pulsatilidade ( $A$ - B / média). Diagnosticou-se aumento de resistência na artéria umbilical quando o IR ou 0 IP encontravam-se acima do percentil 95 para idade gestacional estimada segundo a tabela de Arduini e Rizzo (1990) ${ }^{16}$.

O DV foi identificado em corte transversal ou médio-sagital do abdome superior fetal através do doppler colorido e o local de amostragem foi localizado em sua porção ístmica, mais estreita e com maiores velocidades.

Foram calculadas as velocidades durante sístole ventricular (onda S), durante diástole ventricular (onda D) e sístole atrial (onda $A$ ), 0 índice de pulsatilidade para veias ( $\mathrm{S}$ - A/média) e as relações $S / A$ e $(S-A) / S$.

Após o nascimento, antes do delivramento e mesmo do primeiro movimento respiratório fetal, obteve-se um segmento de $10-20 \mathrm{~cm}$ de comprimento do cordão umbilical por clampeamento com duas pinças. As amostras de sangue (artéria e veia) foram obtidas através de punção (aproximadamente $2 \mathrm{ml}$ ) de ambos os vasos com seringas previamente preparadas.

Foram utilizadas seringas plásticas de $3 \mathrm{ml}$ e agulhas $25 \times 7-22 \mathrm{G}$ descartáveis da marca Becton Dickinson (B-D). Utilizou-se heparina sódica liofilizada em ampolas na concentração de $1.000 \mathrm{UI} / \mathrm{ml}$. A heparina era aspirada para a seringa e homogeneamente distribuída pelas suas paredes. A seguir, o conteúdo era expelido com a seringa posicionada de modo que a agulha localizasse para cima para que 0 ar remanescente fosse eliminado. As amostras eram enviadas imediatamente ao laboratório e analisadas dentro de 5-10 minutos da coleta ${ }^{17}$.

A análise do sangue foi realizada automaticamente pelo aparelho Radiomiter ABL-5. Foram calculados $0 \mathrm{pH}, 0 \mathrm{pCO}{ }_{2}$ e 0 excesso (ou déficit) de base para a artéria e a veia umbilicais.
Considerou-se acidemia valores de pH menores que 7,26 para a veia e 7,20 para a artéria, se a paciente não apresentava contrações uterinas e $\mathrm{pH}<7,20$ para a veia e 7,15 para a artéria umbilical se a paciente apresentou trabalho de parto. Somente foram incluídos como patológicos os RN com diagnóstico de acidemia metabólica ou mista ( $\mathrm{BE}<-10$ e $\mathrm{pCO} \mathrm{O}_{2}>60 \mathrm{mmHg}$ na ausência de trabalho de parto e $\mathrm{BE}<-11$ e $\mathrm{pCO}_{2}^{2}>65 \mathrm{mmH}$ quando contrações uterinas presentes na artéria umbilical ou $\mathrm{BE}<-6$ e pCO $>50 \mathrm{mmH}$ g na ausência de trabalho de parto e $\mathrm{BE}<-8$ e $\mathrm{pCO}_{2}>55 \mathrm{mmHg}$ na presença de contrações uterinas para a veia umbilical ${ }^{2}$. $Q$ uando foi coletado sangue da artéria e da veia umbilical, considerou-se para diagnóstico de acidemia o resultado da artéria umbilical. Somente quando a co leta da artéria não foi efetuada ou a análise foi prejudicada é que foi considerado o resultado obtido na veia umbilical.

As pacientes foram seguidas com exames dopplervelo cimétricos periódicos. A freqüência dos exames foi determinada seguindo protocolos de aco mpanhamento semelhantes em ambosos serviços: levando-se em consideração a condição clínica materna e as alterações encontradas ao doppler. Após a admissão no proto colo de estudo, todas as pacientes permaneceram internadas até o parto. As outras provas de vitalidade fetal também eram realizadas seguindo o mesmo protocolo.

A avaliação do doppler do ducto venoso, no período do estudo, não era critério para resolução da gestação ou mudança de procedimentos no acompanhamento; portanto, foi indiferente o conhecimento ou desconhecimento do resultado desse exame pelo corpo clínico. Q uando solicitado, o resultado não foi omitido.

Os casos não determinados pelos protocolos foram decididos em reuniões científicas com docentes das disciplinas e a chefia da enfermaria de patologia obstétrica.

A análise estatística fo i realizada pelo Departamento de Matemática e Estatística da Universidade Federal do Ceará com o auxílio do programa SPSS $10.0^{\circledR}$ (SPSS C 0, Chicago, IL, USA). Foram calculadas a sensibilidade e a especificidade dos vários pontos de corte das variáveis analisadas no doppler do DV e construída curva ROC para cada parâmetro. Q uando o parâmetro analisado se mostrou bom preditor para 0 diagnóstico de acidemia (área sob a curva significativamente superior a $50 \%$; $p<0,05$ ) selecionou-se o melhor ponto de corte. Para esse valor encontrado foram calculados a sensibilidade, a especificidade, 0 valor preditivo positivo, 0 valor preditivo negativo, a taxa de falsos positivos, a taxa de falsos negativos, 0 valor de predição (acurácia), a razão de verossimilhança positiva e a razão de verossimilhança negativa. As variáveis analisadas no doppler do DV (variáveis independentes) foram: a velocidade durante sístole ventricular (onda S), a velocidade durante diástole ventricular (onda D), a velocidade durante sístole atrial (onda A), o índice de pulsatilidade para veias, a relação $S / A$ e a relação $(S-A) / S$. A variável analisada no nascimento (variável dependente) foi a presença de acidemia metabólica ou mista no cordão umbilical.

\section{RESULTADOS}

Em 19 casos foram colhidas amostras do sangue da veia e da artéria umbilicais no momento do parto, em 26 oportunidades foi 
Carvalho FHC et al.

colhido somente sangue das artérias e em dois casos apenas da veia. Em nenhum dos caso sonde foram examinados ambos os vasos houve diferença no diagnóstico de normalidade ou de acidemia. $0 \mathrm{pH}$ médio da artéria umbilical foi de 7,27 (variando de 7,14 a 7,37) e da veia de 7,27 (variando de 6,97 a 7,41). 0 excesso de base encontrado na artéria umbilical foi, em média, de -5,85 (variando de 0,0 a $-17,0$ ) e na veia de $-6,34$ (variando de 0,0 a - 22,3).

0 bteve-se diagnóstico de acidemia em 12 pacientes $(25,5 \%)$. 0 co rreu acidemia metabólica em seis casos e acidemia mista em seis. $\mathrm{N}$ ão houve diagnóstico de acidemia respiratória.

A análise, através da curva $R O C$, mostrou que a velocidade $S$, a velocidade $D$ e a velocidade $A$ no doppler de DV não se mostraram bons parâmetros para a predição de acidemia no nascimento na população de estudo. A curva ROC para a velo cidade $S$ mostrou área sob a curva de apenas 0,435 [intervalo de confiança $=0,233 ; 0,636$ ] com $p=0,502$. Para a velocidade $D$, a área sob a curva ROC encontradafoi de 0,462 [intervalo de confiança $=0,264 ; 0,660$ ] com $p=0,462$, e a velocidade $A$ mostrou área so b a curva ROC de 0,617 [intervalo de confiança $=0,416 ; 0,817$ ] $\operatorname{com} p=0,232$.

A análise do índice de pulsatilidade para veias do DV na predição de acidemia no nascimento mostra que a área sob a curva ROC foi significativamente superior a $50 \%$, traduzindo esse parâmetro como bom preditor para acidemia. Encontrou-se área so b a curva de 0,790 $[I C=0,624 ; 0,957] \operatorname{com} p=0,003$ (Figura 1 ).

Para decisão do ponto de corte foi construído quadro com as coordenadas da curva ROC determinando a sensibilidade e a taxa de falso-positivo (complementar da especificidade) para os valores encontrados do IPV. Tomou-se como ponto de corte o valor do IPV do DV $=0,76$, onde IPV $>0,76$ implica em maiores chances da presença de acidemia no nascimento. Encontrou-se: sensibilidade de $66,7 \%$, especificidade de $88,6 \%$, valor preditivo positivo (VPP) de $66,7 \%$, valor preditivo negativo (VPN) de $88,6 \%$, taxa de falso-positivo de 33,3\%, taxa de falso-negativo de 11,4\%, acurácia igual a $83 \%$, razão de verossimilhança positiva de 5,8 e razão de verossimilhança negativa de 0,4 .

A análise da relação $S / A$ do $D V$ (considerando $S / A>5,06$, maior valor encontrado, quando a velo cidade A eraigual a zero) na predição de acidemia no nascimento mostra uma área sob a curva ROC significativamente superior a $50 \%$, traduzindo esse parâmetro como bom preditor para acidemia. Encontrou-se uma área sob a curva de $0,818[I C=0,662 ; 0,974] \operatorname{com} p=0,001$ (Figura 2).

0 ponto de corte encontrado para a relação S/A do DV foi 2,67. Calculou-se: sensibilidade de $58,3 \%$, especificidade de $94,3 \%$, VPP de $77,8 \%$, VPN de $86,8 \%$, taxa de falso-positivo de $22,2 \%$, taxa de falso-negativo de $13,2 \%$, acurácia igual a $85,1 \%$, razão de verossimiIhança positiva de 10,2 e razão de verossimilhança negativa de 0,4.

A análise da relação (S - A)/S do DV na predição de acidemia no nascimento mostra uma área sob a curva ROC significativamente superior a 50\%, traduzindo esse parâmetro como bom preditor para acidemia. Encontrou-se uma área sob a curva de 0,818 [IC = 0,662 $; 0,974] \operatorname{com} p=0,001$ (Figura 3).

Encontrou-se como ponto de corte o valor da relação (S - A)/S do $D V=0,63$. Foram calculados: sensibilidade de 58,3\%, especificidade de $94,3 \%$, VPP de $77,8 \%$, VPN de $86,8 \%$, taxa de
Figura 1 - Curva ROC para a análise do índice de pulsatilidade do ducto venoso como diagnóstico da acidemia ao nascimento



Figura 2 - Curva ROC para a análise da relação $S / A$ do ducto venoso como diagnóstico da acidemia no nascimento

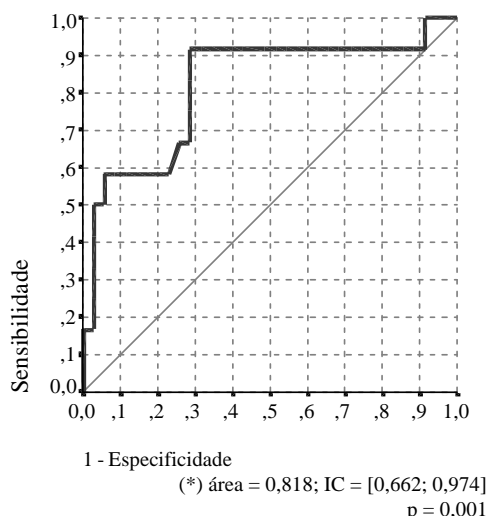

$\mathrm{p}=0,00$

Figura 3 - Curva ROC para a análise da relação (S - A)/S do ducto venoso como diagnóstico da acidemia no nascimento

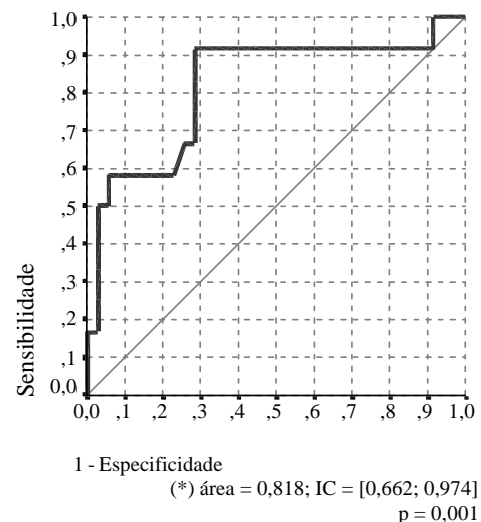

$\mathrm{p}=0,001$ 
falso-positivo de 22,2\%, taxa de falso-negativo de 13,2\%, acurácia igual a 85,1\%, razão de verossimilhança positiva de 10,2 e razão de verossimilhança negativa de 0,4.

0 Teste de MacN emar comparou esses parâmetros entre si e não encontrou diferença estatisticamente significativa na predição de acidemia ao nascimento. Q uando comparou a sensibilidade, encontrou: IPV x relação $S / A, p=1,000 ;$ IPV x $(S-A) / S, p=1,000$ e S/A $x(S-A) / S, p=1,000$. Para a especificidade: IPV $x$ relação $S / A$ encontrou $p=0,500 ; \operatorname{IPV} \times(S-A) / S, p=0,500$ e $S / A \times(S-A) / S$, $p=1,000$. A comparação da acurácia, revelou: IPV x relação $S / A$, $p=1,000 ; \operatorname{IPV} \times(S-A) / S, p=1,000$ e $S / A \times(S-A) / S, p=1,000$.

\section{Discussão}

A insuficiência placentária é uma das principais causas de morbimortalidade perinatal. Sua etiologia é ampla e associa-se freqüentemente com as principais intercorrências clínicas e obstétricas: síndromes hipertensivas, restrição do crescimento fetal, diabetes mellitus, gestação prolongada, colagenoses, síndrome do anticorpo anti-fo sfolípide, cardiopatias, pneumopatias e hemopatias?

0 advento da dopplervelocimetria permitiu 0 estudo da hemodinâmica útero e feto-placentária e proporcionou melho ria nos resultados perinatais nas populações de alto-risco. 0 doppler da artéria umbilical reflete a resistência vascular placentária, fortemente correlacionada com a restrição do crescimento fetal e os efeitos multisistêmicos da deficiência de nutrientes e oxigênio. Por isso, é hoje considerado o melhor método de diagnosticar a insuficiência placentária1, ${ }^{1,2}$.

Este estudo avaliou a relação entre o doppler do DV e a presença de acidemia no nascimento em gestações com diagnóstico de insuficiência placentária pela presença de resistência elevada na artéria umbilical. Foram excluídas outras causas agudas de acidemia fetal, tais como o descolamento prematuro de placenta e a anestesia geral. 0 resultado do doppler venoso não influencio u a decisão da conduta. A época da resolução da gestação foi definida pela idade gestacional, piora do doppler arterial ou padrão patológico na cardio tocografia (CTG) e/ou no perfil biofísico fetal (PBF). 0 resultado do doppler do DV considerado na análise estatística foi realizado a menos de 24 horas do parto, pois alterações podem aparecer rapidamente nos dias que antecedem o padrão anormal na CTG ${ }^{19}$.

0 doppler da artéria umbilical anormal permite a identificação dos fetos de risco para resultados adversos, mas é um pobre preditor da condição fetal. Em contraste, 0 doppler venoso nos permite definir a gravidade dos resultados perinatais, po is relaciona-se com 0 grau do comprometimento circulatório do feto ${ }^{4,7}$.

0 DV parece ser, no momento, o melhor vaso para monitorização de fetos comprometidos colaborando na decisão sobre 0 momento da resolução da gestação, pois alguns autores encontraram que sua anormalidade precede em poucos dias as alterações dos outros testes biofísicos como CTG e PBF ${ }^{9,19}$. No entanto, Baschat (2003) descreveu que a relação temporal na seqüência de deterioração no doppler é mais claramente descrita em fetos prematuros. Próximo ao termo, pode não haver relação dos parâmetros metabólicos com a análise do doppler. Em contraste, a perda dos critérios do PBF com o comprometimento metabólico é independente da idade gestacional ${ }^{4}$.

Do is mecanismos parecem ser responsáveis pelo padrão anormal das ondas de velocidade venosas: um é 0 aumento da pós-carga ventricular direita e 0 outro, a falência mio cárdica ${ }^{20}$. A capacidade de compensação fetal através da centralização está relacionada à adequada oxigenação miocárdica. N esse momento, embora exista aumento da resistência na circulação feto-placentária, a centralização se dásem anormalidades no do ppler venoso. As alterações progressivas na circulação venosa indicam falência no mecanismo compensatório e 0 desenvolvimento de insuficiência cardíaca por hipóxia miocárdica ${ }^{20,21}$. Progressão da anormalidade circulató ria fetal é associada com deterioração do equilíbrio ácido-básico e resultados perinatais adversos. 0 fenômeno da centralização é relacionado com queda da p0 2 no cordão umbilical e padrões anormais de doppler venoso com queda da $\mathrm{p} 02$ mais acentuada, acidemia e morte fetal ${ }^{5}$.

Há na literatura inúmeras investigações demonstrando a predição eficaz de resultados perinatais adversos com 0 doppler venoso, em particular com o DV. No entanto, os estudos que verificaram a predição de acidemia ainda são poucos e não se encontrou consenso sobre qual o melhor parâmetro, e o ponto de corte, para esse fim. A importância de se diagnosticar essa anormalidade é o fato de sabermos que a acidemia metabólica fetal é evento terminal da via que leva os fetos com restrição do crescimento ao óbito ${ }^{19}$.

A análise do sangue do cordão umbilical é considerada o padrão ouro na avaliação da condição bioquímica fetal/neonatal. A aferição dos parâmetros ácido-básicos no cordão umbilical, ao nascimento, mostrou ser mais preciso indicador de asfixia intra-parto que os escores de Apgar, e essa análise deveria ser rotineira no cuidado obstétrico ${ }^{22}$. Alguns pesquisadores sugerem que $0 \mathrm{pH}$ da artéria umbilical reflete com mais acurácia a condição fetal ou neo natal que qualquer outro parâmetro da gasometria arterial ou veno $\mathrm{sa}^{23}$.

0 limite inferior da normalidade para $0 \mathrm{pH}$, abaixo do qual se considera acidemia no nascimento, não foi definido com precisão. O bserva-se variação de 7,00 a 7,2022. Utilizou-se, nessa investigação, os parâmetros de Vintzileos et al. (1992) ${ }^{18}$, que diferenciam o sfetos que se submeteram às contrações uterinas daqueles nascidos por cesariana eletiva. 0 valor do $\mathrm{pH}$ na artéria umbilical, sem trabalho de parto, foi considerado o valor clássico $<7,20.0$ objetivo foi selecionar mais precocemente todos os fetos com acidemia e não apenas aqueles com acidemia grave $(\mathrm{pH}<7,00)$, onde ocorre verdadeira associação com seqüelas neurológicas e, inclusive, morte neonatal. Sabe-se que as acidemias metabólica e mista, diferentemente da acidemia respiratória, são verdadeiramente associadas com complicações neonatais's1.

Encontrou-se 12 RN acidóticos napopulação de estudo $(25,5 \%)$. Freqüência semelhante foi encontrada por Andrade et al. (2002) ${ }^{13}$.

0 s estudos da década de 90 foram realizados com coleta de sangue fetal através de cordocentese. A importância dessas investigações foi mostrar a correlação da acidemia com o doppler de DV anormal; no entanto, essa prática não foi instituída na clínica pelo fato de haver maior risco de complicações nesses fetos já debilitados $5^{3,21,24}$. Rizzo et al. (1996) ${ }^{24}$ encontraram uma área sob a 
curva ROC, para predição de hipoxemia, de 0,66, sensibilidade de $72 \%$ e especificidade de $60 \%$.

$\mathrm{N}$ a literatura nacional, $\mathrm{O}$ IPV é o índice mais freqüentemente testado e, por vezes, encontram-se resultados discordantes. Andrade et al. (2002) $)^{13}$, avaliando gestações de alto risco, encontraram boa correlacão do IPV acima do percentil 95 com acidemia no nascimento: sensibilidade de 39,1\%, especificidade de $90,4 \%$, VPP de $60 \%$ e VPN de $80,2 \%$. Miyadahira (2002) $)^{2}$, no entanto, não encontrou resultados tão animadores: sensibilidade de $36,23 \%$, especificidade de $65,14 \%$, VPP de $15,5 \%$ e VPN de $85,27 \%$. Para 0 último autor, o doppler de DV é um pobre preditor de acidemia. N ossa casuística demonstrou, para o IPV do DV, sensibilidade de $66,7 \%$, especificidade de $88,6 \%$, VPP de $66,7 \%$, VPN de $88,6 \%$ e razão de verossimilhança positiva de 5,8 .

$\mathrm{N}$ a determinação do melhor parâmetro do doppler do DV para diagnóstico de acidemia no nascimento, encontrou-se que as relações $S / A$ e $(S-A) / S$ são igualmente boas, e superiores aos demais, para esse fim. A relação S/A é o índice mais comumente utilizado para descrever o fluxo do DV, segundo Harman e Baschat (2003)7.

0 s pontos de corte encontrados neste estudo, para predição de acidemia, são muito próximos aos calculados por Hofstaetter et al. $(2002)^{15}$ quando avaliaram a predição de mortalidade perinatal. 0 ponto de corte para o IPV encontrado foi de 0,76 (com sensibilidade de $66,7 \%$, especificidade de $88,6 \%$, acurácia de $83 \%$ e área sob a curva ROC de 0,79 ) e para a relação $(S-A) / S$ foi de 0,63 (com sensibilidade de $58,3 \%$, especificidade de $94,3 \%$, acurácia de $85,1 \%$ e área sob a curva ROC de 0,818). N os dados de H ofstaetter et al. os valo res foram, respectivamente, 0,99 (área so b a curva $=0,84$ ) $\mathrm{e}$ 0,70 (área sob a curva $=0,805$ ).

Para a relação S/A encontrou-se ponto de corte de 2,67 (sensibilidade de $58,3 \%$, especificidade de $94,3 \%$ e acurácia de $85,1 \%$ ). Sá et al. $(2003)^{14}$ descreveram como limite superior 0 valor de 3,6 (sensibilidade de $71 \%$, especificidade de $94 \%$ e razão de verossimilhança positiva de 12,14); no entanto, nosso valor coincide com os limites superiores (percentil 95) encontrados por Bahlmann et al. $(2000)^{12}$. Bahlmann et al. verificaram que a relação S/A diminui com a idade gestacional. 0 valor máximo para 32 semanas é 2,791 e para 33 semanas é 2,762. A idade gestacional média da nossa casuística foi 32,9 semanas. Se adotarmos 0 limite de 3,6 descrito por Sá et al. $(2003)^{14}$, encontraremos especificidade de $97 \%$, porém sensibilidade de apenas $25 \%$.

0 valor limite para a relação $(S-A) / S$ de 0,63 também é semelhante aos decritos por Bahlmann et al. $(2000)^{12}$. 0 limite superior na 32a. semanaé 0,631 e na33a semanaé 0,630. Para o IPV encontrou-se, nesta casuística, valor pouco inferior aos limites descritos por aqueles autores. Eles descreveram como limites máximos para 32 semanas 0,977 e para 33 semanas 0,974.

As velocidades $S$ e $D$ no doppler do DV geralmente pouco se alteram em fetos comprometidos e oferecem poucas possibilidades para diagnóstico. Não se verificou, nesse estudo, benefício dessas velocidades na predição de acidemia. Surpreendente foi 0 achado que a velocidade A não se mostrou boa preditora de acidemia, pois se sabe que ela declina à medida que progride 0 comprometimento fetal. Hofstaetter et al. (2002) ${ }^{15}$ encontraram boa predição dessa velocidade para mortalidade perinatal. $\mathrm{A}$ área sob a curva $\mathrm{ROC}$ encontrada foi de 0,788. Para o ponto de corte descrito de $>17 \mathrm{~cm} / \mathrm{s}$, a sensibilidade encontrada foi de $79 \%$ e a especificidade de $68 \%$.

Francisco $(2002)^{25}$ demonstrou que o doppler do DV foi o único teste capaz de predizer acidemia em gestações com diástole zero ou reversa na artéria umbilical. Foi adotado como ponto de corte 0 percentil 95 do IPV para idade gestacional. 0 IPV médio nos fetos com pH $<7,20$ foi 1,19 e nos casos com pH = 7,20 foi 0,82. A freqüência de acidemia no último grupo foi de $48,4 \%$.

Vários autores descreveram que 0 doppler do DV precede as alterações dos testes tradicionais de avaliação do bem-estar fetal, CTG e PBF, em fetos prematuro $5^{4,9,19}$. Apesar das evidências, aépoca da resolução da gestação ainda é definida em muitos serviços pelos traçados anormais da freqüência cardíaca fetal. Porém, nesse momento, mais de $64 \%$ dos fetos estão extremamente hipoxemiados e acidóticos ${ }^{11}$.

0 doppler de DV mostrou eficácia em predizer acidemia nas gestações com insuficiência placentária. 0 utro dado a ser observado é que se trata de teste específico, demonstrando a sua importância na to mada de decisão quando se pesa o risco da prematuridade com os riscos de comprometimento fetal e óbito intra-uterino nessa situação. Deve ser adicionado ao arsenal propedêutico no seguimento de fetos com diagnóstico de alteração no território arterial. Pode ajudar a diminuir as taxas de falso-positivo da CTG que possui boa sensibilidade, porém baixa especificidade.

Decisão de interrupção da gestação, na presença de doppler venoso anormal, poderia ser sugerida pela inegável associação com acidemia encontrada nesse estudo e em dados da literatura, ${ }^{5,15}$. N 0 entanto, é necessário cautela antes da generalização, po is as correlações mais evidentes são com o estado fetal (equilíbrio ácidobásico, natimortalidade e sofrimento fetal). As associações com as complicações neo natais são menos evidentese não foram testadas no presente estudo. Isso indica que a condição neonatal é sujeita a muitas variáveis, das quais a idade gestacional no parto parece ser a mais aparente 5 . 0 benefício da adição do doppler de DV na avaliação só pode ser calculado em investigações prospectivas e randomizadas que incluam a condição materna, a idade gestacional e o poder preditivo das anormalidades específicas das outras provas de bem-estar fetal, 0 que torna 0 estudo complicado. Portanto, mais pesquisas são necessárias para delinear os protocolos de acompanhamento perinatal, com os atuais e futuros conhecimentos, dessas gestações que portam um problema tão complexo quanto a insuficiência placentária.

\section{CONCLUSÖES}

O síndicesângulo-independentes do doppler do DV mostraramse bons preditores de acidemia ao nascimento em gestações com insuficiência placentária. As relações $S / A$ e $(S-A) / S$ e 0 índice de pulsatilidade do DV mostraram-se estatisticamente similares nesta predição. Os pontos de corte determinados para os parâmetros do doppler do DV foram: IPV $=0,76$, relação $S / A=2,67$ e $(S-A) / S=0,63$.

Conflito de interesse: não há. 


\section{SUMMMARY}

\section{Ductus Venosus Doppler Velocimetry to predict acidemia AT BIRTH IN PREGNANCIES WITH PLACENTAL INSUFFCIENCY}

O BJETIVES. To investigate the po ssibility of predicting acidemia at birth in pregnancies with placental insufficiency by Doppler velocimetry of the ductus venosus and to establish the best parameter and cut-off points in this prediction.

METH ODS. This was a prospective cross-sectional study, involving 47 single pregnancies with placental insufficiencyafter 26 w eeks of gestation, carried out at the "H o spital São Paulo (UN IFESP) and M aternidade-Escola Assis Chateaubriand (UFC)". Placental insufficiency was defined as the umbilical artery pulsatility index above the $95^{\text {th }}$ percentile for gestational age. Fetuses with chromosomal or structural anomalies were excluded. Thetime interval between the Doppler velocimetryand the birth was ofless than 24 hours. The umbilical arterial blood samples were collected immediately after birth. Acidemia was defined as umbilical arterial pH $<$ 7.2 in the absence of uterine contractions and $<7.15$ in the presence of contractions. Metabolic or mixed acidemia at birth was considered pathological. Receiver operating characteristics (ROC) curves were calculated for $S, D$ and $A$-velocities, pulsatility index for veins and the $S / A$ ratio and (S-A)/Sratio of the ductusvenosus. Parameters were compared usingthe MacN emarTest

RESULTS. S, D and A-velocities of the ductus venosus were poor predictors of acidemia at birth. The pulsatilityindex for veins (area under the curve $0.79, p=0.003$ ), $S / A$ ratio and $(S-A) / S$ ratio (area under the curve $0.818, p=0.001$ ) of the DV were strongly related to fetal acidemia. The cut-off points calculated were: pulsatility index for veins $=0.76 ; \mathrm{S} / \mathrm{A}$ ratio $=2.67$ and $(S-A) / S$ ratio $=0.63$.

CONCLUSION S. The angle-independent indices of the DVDoppler are adequate for the diagnosis of fetal acidemia in gestations with placental insufficiency. No statistically significant differences were observed between these parameters. [Rev Asso c M ed Bras 2005; 51(4): 221-7]

\section{KEY wO RDS: D oppler velocimetry. Ductus venosus. Fetal acidemia.}

\section{REFERÊNCIAS}

1. Pardi G, Marconi AM, Cetin I. Placental-fetal interrelationship in IU GR fetuses: a review. Placenta 2002;23:S136-41.

2. Miyadahira S. Avaliação da função placentária por meio da dopplervelo cimetria das artérias umbilciais: relação com os resultados dos exames de avaliação da vitalidade fetal e com os pós-natais [livre docência]. São Paulo: Universidade de São Paulo; 2002.

3. Hecher K, Campbell S, D oyle P, H arrington K, N icolaides K. Assessment of fetal compromise by Doppler ultrasound investigation of fetal circulation. Arterial, intracardiac, and venous blood flow velocity studies. Circulation 1995;91:129-38.

4. Baschat AA. Integrated fetal testing in growth restriction: combining multivessel doppler and biophysical parameters. Ultrasound 0 bstet Gynecol 2003;21:1-8.

5. Baschat AA, Gembruch U, Reiss I, Gortner L, W einer CP, Harman CR. Relationship between arterial and venous $D$ oppler and perinatal outcome in fetal growth restriction. U Itrasound O bstet Gynecol 2000;16:407-13.

6. Romero R, Kalache KD, Kadar N. Timing the delivery of the preterm severely growth-restricted fetus: venous D oppler, cardiotocography or the biophysical profile? U Itrasound 0 bstet Gynecol 2002;19:118-21.

7. Harman CR, Baschat AA. Arterial and venous Dopplers in IUGR. Clin O bstet $G$ ynecol 2003;46:931-46.
8. Kiserud T. The ductus venosus. Semin Perinatol 2001;25:11-20.

9. Harrington KF. Making best and appropriate use of fetal biophysical and Doppler ultrasound data in the management of the growth restricted fetus. U Itrasound O bstet Gynecol 2000;16:399-401.

10. Kiserud T, Eik-N es SH, Blaas HG, Hellevik LR, Simensen B. Ductus venosus blood velocity and the umbilical circulation in the seriously growth-retarded fetus. Ultrasound O bstet Gynecol 1994;4:109-14.

11. O zcan T, Sbracia M, d'Ancona RL, Copel JA, Mari G. Arterial and venous Doppler velocimetry in the severely growth-restricted fetus and asso ciations with adverse perinatal outcome. $\mathrm{U}$ Itrasound $\mathrm{O}$ bstet $\mathrm{G}$ ynecol 1998;12:39-44.

12. Bahlmann F, W ellek S, Reinhardt I, Merz E, Steiner E, W elter C. Reference values of ductus venosus flow velocities and calculated waveform indices. Prenat D iagn 2000;20:623-34.

13. Andrade JQ, Miyadahira S, N omura RMY, Francisco RPV, Zugaib M. D opplervelocimetria dos compartimentos arterial e venoso da circulação fetal e umbilical em gestação de alto-risco. Análise dos resultados perinatais. Rev Bras G inecol O bstet 2002;24:153-60.

14. Sá RAM, Netto HC, Lopes LM, Barreto MJV, Cabral ACV. $D$ opplerfluxometria do ducto venoso: relação com a gasometria em fetos prematuros com centralização de fluxo sangüíneo. Rev Bras Ginecol O bstet 2003;25:261-8.

15. Hofstaetter C, Gudmundsson S, Hansmann M. Venous Doppler velocimetry in the surveillance of severely compromised fetuses. U Itrasound O bstet G ynecol 2002;20:233-9.

16. Arduini D, Rizzo G. N ormal values of pulsatility index from fetal vessels: a cross-sectional study on 1556 healthy fetuses. J Perinatol Med 1990;18:165-72.

17. Riley RJ, Johnson JW C. Colleting and analyzing cord blood gases. Clin O bstet Gynecol 1993;36:13-23.

18. Vintzileos AM, Egan JFX, C ampbell W A, Rodis JF, Scorza WE, Fleming AD, et al. Asphyxia at birth as determined by cord blood pH measurements in preterm and term gestations: correlation w ith neo natal outcome. J Matern Fetal Med 1992;1:7-13.

19. Ferrazzi E, Bozzo M, Rigano S, Bellotti M, Morabito A, Pardi G, et al. Temporal sequence of abnormal D oppler changes in the peripheral and central circulatory systems of the severely growth-restricted fetus. U Itrasound O bstet Gynecol 2002;19:140-6.

20. Hecher K, Campbell S. Characteristics of fetal venous blood flow under normal circumstances and during fetal disease. Ultrasound $\mathrm{O}$ bstet Gynecol 1996;7:68-83.

21. Rizzo G, Capponi A, Arduini D, Romanini C. The value of fetal arterial, cardiac and venous flows in predicting $\mathrm{pH}$ and blood gases measures in umbilical blood at cardocentesis in growth retarded fetuses. $\mathrm{Br} J \mathrm{O}$ bstet Gynaecol 1995;102:963-9.

22. ACOG. U mbilical artery blood acid-base analysis. ACOG Technical Bulletin N umber 216; 1995. Int J Gynaecol O bstet 1996;52:305-10.

23. G ordon A, Johnson JW C. Value of umbilical blood acid-base studies in fetal assessment. J Reprod Med 1985;30:329-36.

24. Rizzo G, Capponi A, Talone PE, Arduini D, Romanini C. D oppler indices from inferior vena cava and ductus venosus in predicting $\mathrm{pH}$ and oxygen tension in umbilical blood at cordocentesis in growth-retarded fetuses. U Itrasound O bstet Gynecol 1996;7:401-10.

25. Francisco RPV. Predição de valores de $\mathrm{pH}$ e de déficit de bases no nascimento em gestações com diástole zero ou reversa à dopplervelocimetria das artérias umbilicais [tese]. São Paulo: U niversidade de São Paulo; 2002.

Artigo recebido: 14/04/04

Aceito para publicação: 04/08/04 